\title{
Effect of Different Approaches to Decouple the Dependence of Nuclei-Liquid Surface Energy on Size and Temperature
}

\author{
Mara Cristina Freitas ${ }^{\mathrm{a}}$, Dalmo Inácio Galdez Costa ${ }^{\mathrm{a}}$, Aluísio Alves Cabral ${ }^{\text {**, }}$ \\ Adalto Rodrigues Gomes ${ }^{\mathrm{a}}$,José Manuel Rivas Mercury ${ }^{\mathrm{a}, \mathrm{c}}$ \\ ${ }^{a}$ Department of Mechanics and Materials - DMM - CEFET - MA, Brazil \\ ${ }^{\mathrm{b}}$ Department of Exact Sciences - DCE - CEFET - MA, Brazil \\ ${ }^{\mathrm{c}}$ Academic Department of Chemistry - DAQ - CEFET - MA, Brazil
}

Received: October 27, 2008; Revised: February 14, 2009

\begin{abstract}
Tests involving the Classical Nucleation Theory (CNT) often disregard the size dependence of surface energy. Thus, the surface energy of critical nuclei is assumed to be a macroscopic quantity that depends only on the temperature of a flat surface. However, because the size of critical nuclei changes with temperature, $\sigma_{\mathrm{cl}}(\mathrm{T})$ should be described as a function of both temperature and size of critical nuclei. The present work examines the temperature dependence of macroscopic surface energy, decoupling it from the size dependent part. Tolman, Rasmussen and Vogelsberger's equations are used to decouple the dependence of surface energy on size, using experimental data for the following silicate glasses $\mathrm{Li}_{2} \mathrm{O} \cdot 2 \mathrm{SiO}_{2}\left(\mathrm{LS}_{2}\right)$ and $\mathrm{Na}_{2} \mathrm{O} \cdot 2 \mathrm{CaO} \cdot 3 \mathrm{SiO}_{2}\left(\mathrm{~N}_{1} \mathrm{C}_{2} \mathrm{~S}_{3}\right)$. These equations are successful in obtaining a decrease in $\sigma_{\mathrm{cl}}(\mathrm{T})$, in agreement with theoretical predictions. For all the values of $\delta$, Tolman's equation produces the lowest values of $\sigma_{\mathrm{cl}}(\mathrm{T})$. Nevertheless, they are very close to the liquid/ vapor surface energy $\left(\sigma_{\mathrm{lv}}\right)$, which contradicts the Stefan's rule (i.e. $\sigma_{\mathrm{cl}} / \sigma_{\mathrm{lv}}<<1$ ). Therefore, it is demonstrated that the assumption of the curvature dependence of surface energy does not suffice, per se, to explain the discrepancy between the experimental and theoretical values of nucleation rates.
\end{abstract}

Keywords: crystal nucleation kinetics, nuclei-liquid surface energy, decoupling equations

\section{Introduction}

The Classical Nucleation Theory (CNT) is well known for its good description of the temperature dependence of the nucleation rate, I(T). Nevertheless, CNT tests involving several silicate glasses that nucleate homogeneously have demonstrated that experimental crystal nucleation rates are much higher than theoretical ones ${ }^{1-5}$. These tests consist of plotting $\mathrm{Ln}(\mathrm{I} \eta / \mathrm{T}) \mathrm{vs} .1 / \mathrm{T} \Delta \mathrm{G}_{\mathrm{v}}^{2}$, where the intercepts and slopes are proportional, respectively, to the pre-experimental factor and the surface energy of a flat interface. According to Cabral $\mathrm{Jr}^{6}$, this discrepancy persists even if different free energy expressions are used. These tests are often based on the assumption that surface energy does not change as a function of temperature and nucleus size.

Several assumptions have been investigated to explain the reasons for these discrepancies: i) metastable phase formation ${ }^{7-8}$; ii) the influence of $\mathrm{H}_{2} \mathrm{O}$ content on crystal nucleation rates ${ }^{9}$; iii) the possible breakdown of the Stokes-Einstein equation at $\left(\Delta \mathrm{T} / \mathrm{T}_{\mathrm{m}} \sim 0.5-0.6\right)$, which must not be applied to express the crystal nucleation kinetic as governed by viscous flow ${ }^{10}$; and iv) the absence of an experimental technique to accurately determine the surface energy of nanometric aggregates independently of nucleation experiments ${ }^{11}$. Based on the latter assumption, the CNT assumes that surface energy is a macroscopic thermodynamic property with a value equal to that of a planar interface, $\sigma_{\infty}$. In other words, surface energy is considered to be independent of nucleus size. This assumption is known as the capillarity approximation.

This discrepancy can be avoided by calculating a specific surface energy from experimental nucleation rate data at each temperature, using the theoretical value of the pre-exponential term. In this case, an increase in the temperature dependence of surface energy has been observed, as demonstrated by Turnbull ${ }^{12}$, Spaepen ${ }^{13}$ and James ${ }^{1}$.

On the other hand, Gutzow et al. ${ }^{14}$ demonstrated that the crystalmelt surface energy, $\sigma_{\mathrm{cl}}$, should decrease with increasing temperature, mainly in cases where the molar volume of the liquid phase is higher than the corresponding one of the crystal phase. In addition, there is some experimental evidence that crystal-melt surface energy should decrease with increasing temperature ${ }^{11}$.

According to several researchers ${ }^{16-17}$, a plot of $\sigma_{\mathrm{cl}}(\mathrm{T})$ may indicate a size effect, because the surface energy $\sigma_{c l}(T, R)$ calculated from nucleation data refers to nuclei of critical size, $R^{*}$. The latter parameter changes as a function of temperature.

Recently, Fokin \& Zanotto ${ }^{11}$ applied the Tolman equation to decouple the temperature and size-dependent parts of surface energy from the homogeneous crystal nucleation kinetics of $\mathrm{Li}_{2} \mathrm{O} .2 \mathrm{SiO}_{2}\left(\mathrm{LS}_{2}\right)$ and $\mathrm{Na}_{2} \mathrm{O} .2 \mathrm{CaO} .3 \mathrm{SiO}_{2}\left(\mathrm{~N}_{1} \mathrm{C}_{2} \mathrm{~S}_{3}\right)$ stoichiometric silicate glasses. Based on experimental data of crystal nucleation rates, viscosity, and induction time, and on the difference between the volume free energies of glass and crystal, the authors demonstrated that the surface tension can decrease with temperature, since reasonable values were chosen for the Tolman parameter.

Taking into account the curvature dependence of surface tension, this paper focuses on the effects of the application of different expressions described in the literature on the decoupling of the temperature and size parts of surface energy. In addition to Tolman's approach ${ }^{18}$, the expressions derived by Vogelsberger ${ }^{15}$ and Rasmussen ${ }^{19}$ were also applied to the crystal nucleation kinetics of $\mathrm{LS}_{2}$ and $\mathrm{N}_{1} \mathrm{C}_{2} \mathrm{~S}_{3}$ silicate glasses to compare them with the $\sigma_{\mathrm{cl}}(\mathrm{T})$ results obtained by Fokin $\&$ Zanotto $^{11}$ using only Tolman's equation.

\section{Theory}

According to the Classical Nucleation Theory (CNT), the steadystate homogeneous nucleation rate $\left(\mathrm{I}_{\mathrm{st}}\right)$ can be described as a function of temperature through the following expression ${ }^{12}$ : 


$$
I_{s t}=A \cdot \exp \left[-\frac{\left(W^{*}+\Delta G_{D}\right)}{k \cdot T}\right]
$$

where $I_{\text {st }}$ represents the number of nuclei formed per unit volume $\left(\mathrm{m}^{-3} / \mathrm{s}\right), \mathrm{A}$ is a weakly temperature-dependent term, $\mathrm{k}$ is Boltzmann's constant, $\Delta \mathrm{G}_{\mathrm{D}}(\mathrm{J} / \mathrm{mol})$ is the kinetic barrier for nucleation (which corresponds to the activation energy required to transport a structural unit through the nuclei/glass interface), and $\mathrm{W}^{*}(\mathrm{~J} / \mathrm{mol})$ is the thermodynamic barrier for the formation of critical size nuclei.

The pre-exponential term A is given by:

$$
A=2 N v_{o}\left(\frac{\lambda^{2} \sigma_{\infty}}{k T}\right)^{1 / 2}
$$

where $\mathrm{N}$ is the number of molecules with size $\lambda$ per unit of volume, $v_{0}$ is the vibration frequency of a structural unit for typical nucleation temperatures, $\sigma_{\infty}$ corresponds to the free energy per unit area of crystal/melt flat interface, and h is Planck's constant.

For spherical nuclei, $\mathrm{W}^{*}$ is given by:

$$
W^{*}=\frac{16 \pi \sigma_{\infty}^{3}}{3 \Delta G_{v}^{2}}
$$

where $\Delta \mathrm{G}_{\mathrm{v}}\left(\Delta \mathrm{G}_{\mathrm{v}}=\Delta \mathrm{G} / \mathrm{V}_{\mathrm{m}}\right)$ is the free energy change per unit volume of crystal, $\Delta \mathrm{G}$ is the free energy change per mole, and $\mathrm{V}_{\mathrm{m}}$ is the molar volume of the crystalline phase.

If one neglects a possible breakdown of the Stokes-Einstein equation at deep undercooling $\left(\Delta \mathrm{T} / \mathrm{T}_{\mathrm{m}} \sim 0.5-0.6\right)$ and expresses the kinetic barrier in terms of viscosity, Equation (1) can be rewritten as:

$$
I_{s t}=\frac{2(k T \sigma)^{1 / 2}}{\lambda^{5} \eta} \exp \left(-W^{*} / k T\right)
$$

If the kinetic barrier of nucleation, $\Delta \mathrm{G}_{\mathrm{D}}$, is expressed in terms of the induction period of nucleation, $t_{\text {ind }}$, Equation (1) can be transformed into Equation (5):

$$
T_{s t}=\frac{16 \pi N_{i}^{2}(k T)^{1 / 2} \sigma^{3 / 2}}{3 \Delta G_{v}^{2} t_{i n d}} \exp \left(-\frac{16 \pi V_{m}^{2} \sigma_{\infty}^{3}}{3 \Delta G_{v}^{2} k T}\right)
$$

From Equation (1), one can see that the crystal nucleation rates can be strongly influenced by the values of $\mathrm{W}^{*}$. Therefore, if one neglects the strain energy associated with the formation of critical nuclei, the overall thermodynamic work, W, can be written as:

$$
\mathrm{W}=4 / 3 \pi \mathrm{R}^{3} \Delta \mathrm{G}_{\mathrm{v}}-4 \pi \mathrm{R}^{2} \sigma
$$

Several approximated equations have been derived to describe the curvature dependence of the crystal-melt surface energy, $\sigma=\sigma_{\mathrm{cl}}(\mathrm{R})$ :

$$
\begin{aligned}
& \sigma_{c l}(R)=\frac{\sigma_{\infty}}{1+2 \delta / R}, \text { Tolman } \\
& \sigma_{c l}(R)=\sigma_{\infty}(1-2 \delta / R), \text { Vogelsberger } \\
& \sigma_{c l}(R)=\sigma_{\infty}(1-\delta / R)^{2}, \text { Rasmussen }
\end{aligned}
$$

where Tolman's parameter, $\delta$, characterizes the width of the interfacial region between the coexisting phases (whose order is of atomic dimensions).

It should be noted that these decoupling equations comprise a large range of $\sigma_{\mathrm{cl}}(\mathrm{R})$ values. Nevertheless, Schmelzer et al. ${ }^{15}$ have demonstrated that the Tolman's (Equation (7)), Vogelsberger's (Equation (8)) and Rasmussen's (Equation (9)) expressions can be applied only to $R>>\delta, R>>4 \delta$ and $R>>3 \delta$, respectively. It should be noted that the negative and infinitive values of $\sigma$ obtained for small values of $\mathrm{R} / \delta$ can be neglected.

\section{Calculations}

Experimental data of crystal nucleation rates, viscosity, induction time and thermodynamic quantities for the $\mathrm{LS}_{2}$ and $\mathrm{N}_{1} \mathrm{C}_{2} \mathrm{~S}_{3}$ silicate glasses were selected from the literature, as indicated in Table 1.

The same values of $\delta$ used by Fokin $\&$ Zanotto $^{11}$ were also used here to evaluate $\sigma_{\mathrm{cl}}(\mathrm{R}, \mathrm{T})$ through the decoupling equations for the silicate glasses investigated.

By combining Equation (6) and (7), one obtains:

$$
W=\frac{4}{3} \pi R^{3} \Delta G_{v}-4 \pi R^{3} \frac{\sigma_{\infty}}{(R+2 \delta)}
$$

From the condition $(\partial \mathrm{W} / \partial \mathrm{R})_{\mathrm{R}^{*}}=0$, and taking the positive root, one can find the critical radius as:

$$
R^{*}=\frac{-2 \Delta G_{v} \delta+\sigma_{\infty}+\sqrt{2 \Delta G_{v} \delta \sigma_{\infty}+\sigma_{\infty}^{2}}}{\Delta G_{v}}
$$

The corresponding thermodynamic barrier for nucleation $\left(\mathrm{R}>\mathrm{R}^{*}\right)$ can then be described as:

$$
\begin{aligned}
& W^{*}=-\frac{\pi\left(-4 \Delta G_{v} \delta+2 \sqrt{2 \Delta G_{v} \delta \sigma_{\infty}+\sigma_{\infty}^{2}}\right)^{3}}{6 \Delta G_{v}^{2}}+ \\
& \frac{\pi\left(-4 \Delta G_{v} \delta+2 \sigma_{\infty}+2 \sqrt{2 \Delta G_{v} \delta \sigma_{\infty}+\sigma_{\infty}^{2}}\right)^{2} \sigma_{\infty}}{6 \Delta G_{v}^{2}\left(1+\frac{4 \delta G_{v}}{-4 \Delta G_{v} \delta+2 \sigma_{\infty}+2 \sqrt{2 \Delta G_{v} \delta \sigma_{\infty}+\sigma_{\infty}^{2}}}\right)}
\end{aligned}
$$

If one replaces Equation (12) with (4) or (5), only two parameters, $\sigma_{¥}$ and $\mathrm{d}$, will remain unknown in the resulting equation. In possession of all the experimental data - crystal nucleation rates, viscosity, induction time and difference in free energy per unit of volume - for each silicate glass investigated and at fixed values of $\delta$, one can then determine the $\sigma(\mathrm{T})$ dependence at different values of $\delta$.

Similar procedures were employed to obtain the $\sigma_{\mathrm{cl}}(\mathrm{T})$ curves using the other decoupling equations (Equation (8) and(9)).

\section{Results and Discussion}

If one takes into account the dependence of surface tension on curvature, a significant quantitative change is expected in the work of critical cluster formation, as indicated in Figure 1. The typical values of the parameters used to calculate $\mathrm{W}$ as a function of the nucleus size for different approximations of $\sigma_{\mathrm{cl}}(\mathrm{R})$ are given in the caption of Figure 1.

It should be emphasized that to determine accurately the work of critical cluster formation, one should take into account the dependence of nuclei density on size, $\rho(R)^{23}$. However, simulations carried out by Fokin and Zanotto ${ }^{11}$ for a model glass demonstrated that $\mathrm{W}^{*}$ is weakly affected by $\rho(R)$.

Additionally, as the temperature changes, one also expects a decrease in crystal density with temperature, $\rho(T)$. However, the

Table 1. Sources of experimental data used to compute $\sigma(\mathrm{T})$.

\begin{tabular}{lcccccc}
\hline Glass & $\mathrm{I}$ & $\eta$ & $\tau$ & $\Delta \mathrm{G}_{\mathrm{v}}$ & $\mathrm{V}_{\mathrm{m}}$ & $\lambda$ \\
\hline $\mathrm{LS}_{2}$ & {$[11]$} & {$[11]$} & {$[11]$} & {$[11]$} & {$[6]$} & {$[6]$} \\
$\mathrm{N}_{1} \mathrm{CS}_{3}$ & {$[20]$} & {$[20]$} & {$[20]$} & {$[20]$} & {$[6]$} & {$[6]$} \\
\hline
\end{tabular}




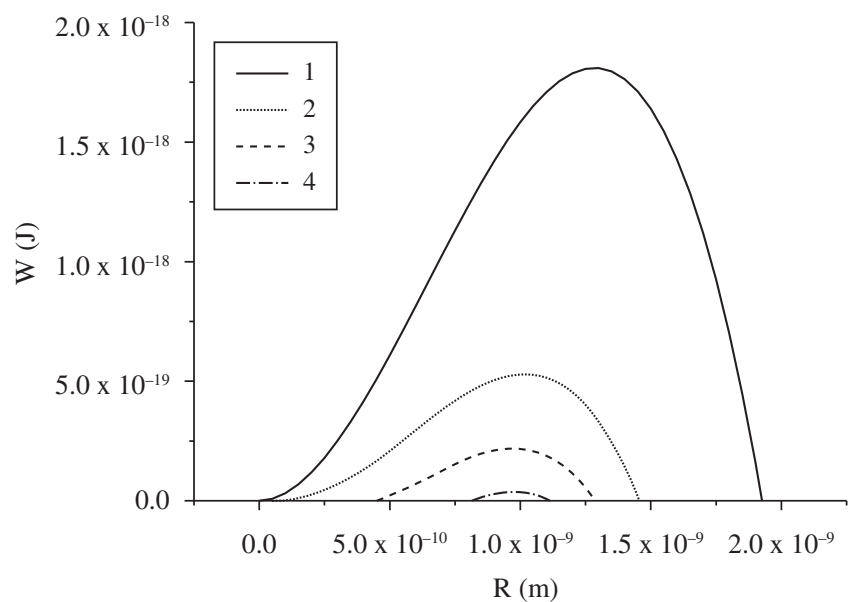

Figure 1. Calculated free energy changing of a model system due to the formation of nucleus as a function of size: (1) $\sigma$ is size independent; (2) $\sigma$ is size dependent (Equation (7)); (3) $\sigma$ is size dependent (Equation (8)); (4) $\sigma$ is size dependent (Equation (9)). The following parameters were used: $\Delta \mathrm{G}_{\mathrm{v}}=4.08 \times 10^{8} \mathrm{~J} / \mathrm{m}^{3}, \sigma_{\infty}=0.262 \mathrm{~J} . \mathrm{m}^{-2}$.

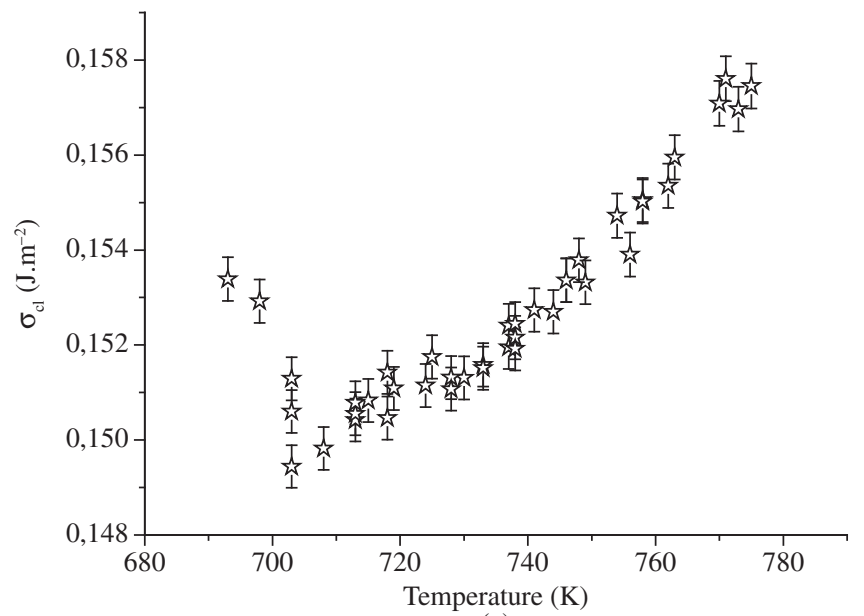

(a)

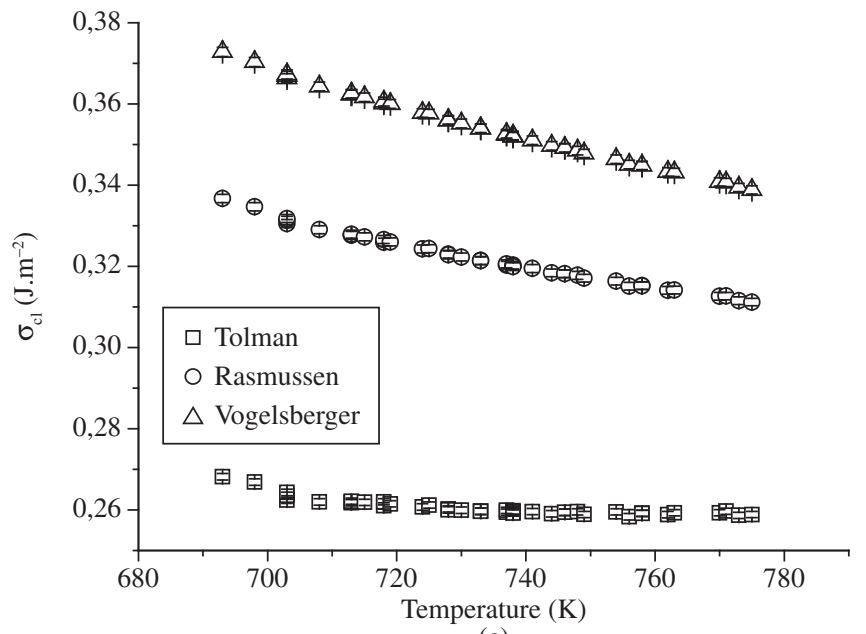

(c) results obtained by Fokin and Zanotto ${ }^{11}$ for a $\mathrm{LS}_{2}$ glass demonstrated that $\sigma_{\mathrm{cl}}(\mathrm{T})$ is weakly affected by $\rho(\mathrm{T})$.

Hence, the calculations of $\sigma_{\mathrm{cl}}(\mathrm{R}, \mathrm{T})$ presented in this paper disregarded the effects of $\rho(R)$ and $\rho(T)$. Figures 2 and 3 show the $\sigma_{c l}$ vs. T curves obtained for the $\mathrm{LS}_{2}$ and $\mathrm{N}_{1} \mathrm{C}_{2} \mathrm{~S}_{3}$ glasses using viscosity data. The error bars indicated in each figure are between 0.3 and $1 \%$. As expected, the $\sigma_{\mathrm{cl}}(\mathrm{T})$ plots obtained from experimental induction time data showed a behavior similar to that depicted in Figures 2 and 3 of this paper. Nevertheless, only the $\sigma_{\mathrm{cl}}(\mathrm{T})$ curves obtained from viscosity data are presented on Figures 2 and 3.

As can be seen in Figures 2 and 3, the $\sigma_{\mathrm{cl}}(\mathrm{R}, \mathrm{T})$ curves calculated from viscosity follow the same tendency, i.e., $\sigma_{\text {cl(Tolman) }}<\sigma_{\text {cl(Rasmussen) }}$ $<\sigma_{\mathrm{cl}(\mathrm{Vogelsberger})}$. This behavior is similar to that obtained by using the induction time as the kinetic barrier.

At temperatures below $\mathrm{T}_{\mathrm{g}}$, it is well known that the elastic strain resulting from the difference between the densities of glass and crystal can underestimate the driving force for crystallization and, hence, overestimate the thermodynamic barrier for nucleation, $\mathrm{W}^{* 22}$. Therefore, the slopes of the $\sigma_{\mathrm{cl}}(\mathrm{T})$ curves were estimated from temperatures higher than $\mathrm{T}_{\mathrm{g}}$. Figures 4 and 5 illustrate the behavior of

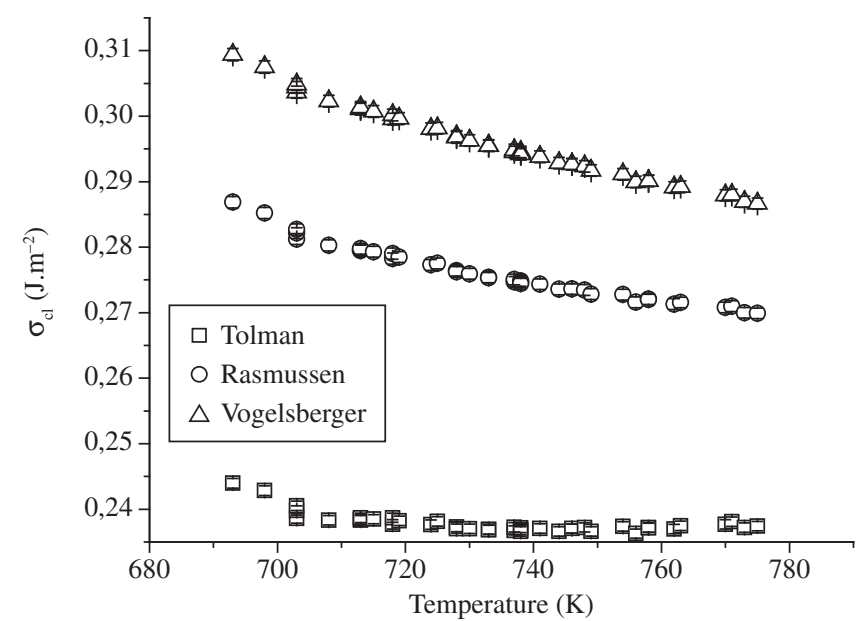

(b)

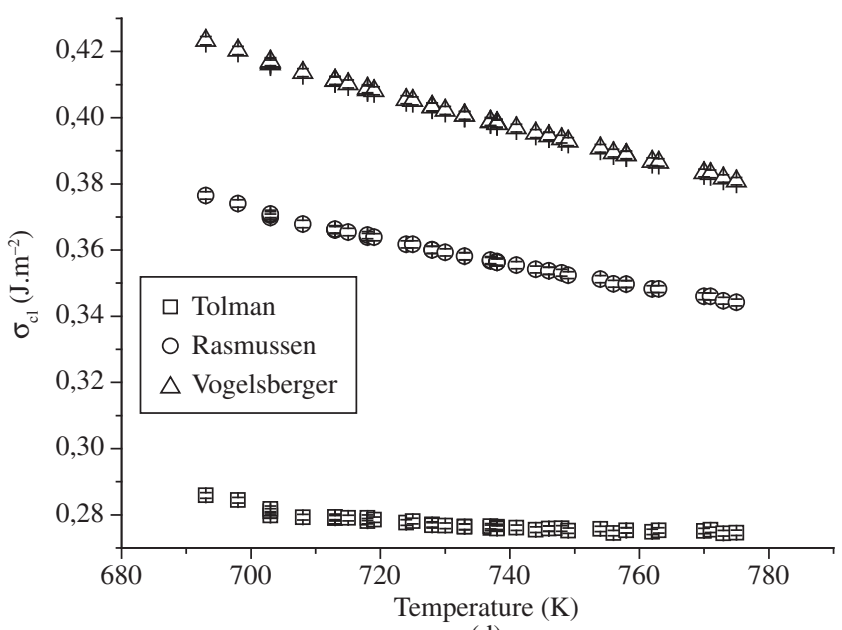

(d)

Figure 2. Crystal-melt surface energy as a function of the temperature calculated for a $\mathrm{LS}_{2}$ glass, using viscosity data and the equations proposed by Tolman (口), Rasmussen ( $\left(\right.$ ) and Vogelsberger $(\triangle)$. The $\delta$ values used were: a) 0 ; b) $2.33 \times 10^{-10} \mathrm{~m}$; c) $3 \times 10^{-10} \mathrm{~m}$; d) $3.5 \times 10^{-10} \mathrm{~m}$. For $\delta=0$, the same values of $\sigma_{\mathrm{c}}$ were obtained for all decoupling equations. These values are indicated as $(\star 2)$. 

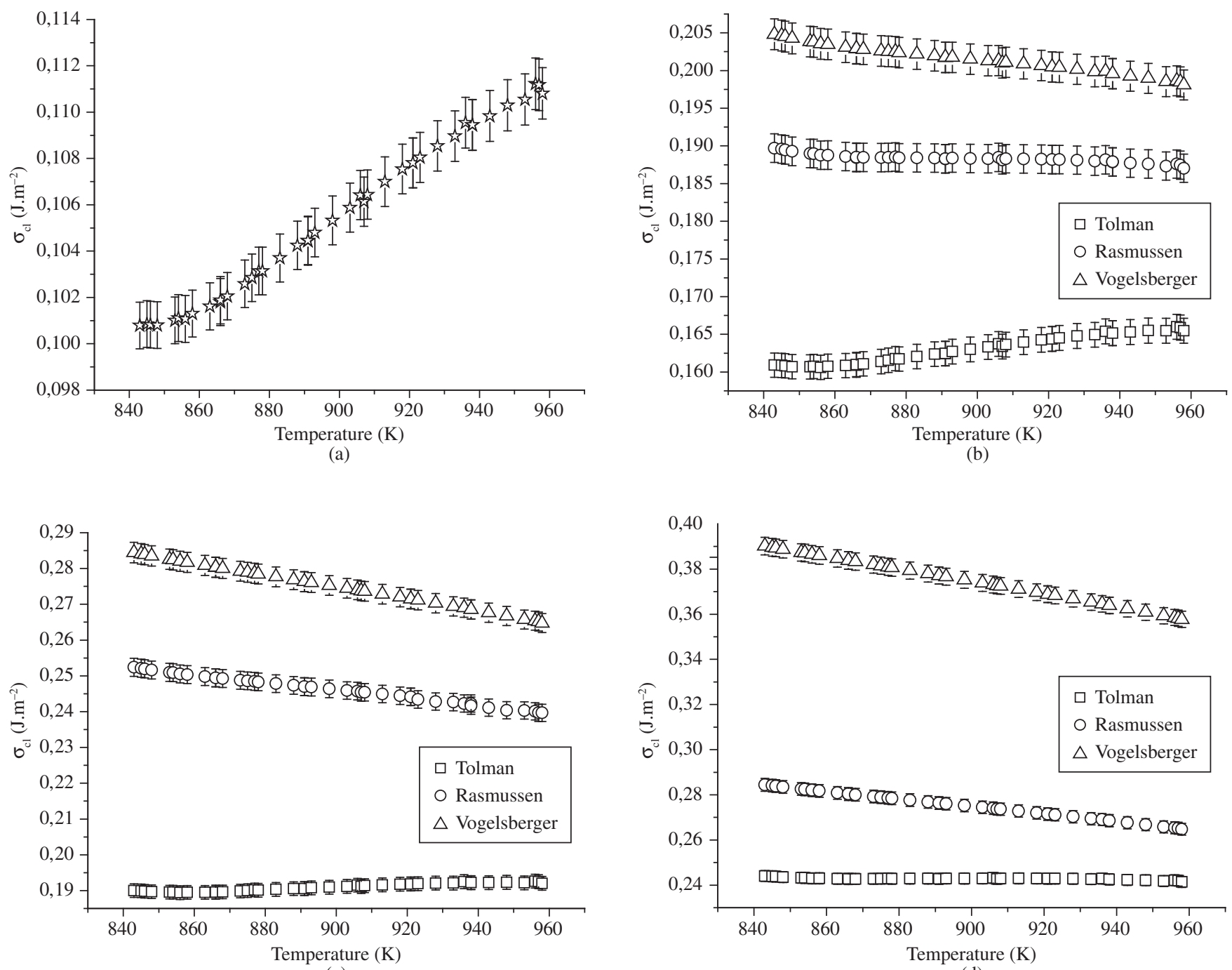

(c)

(d)
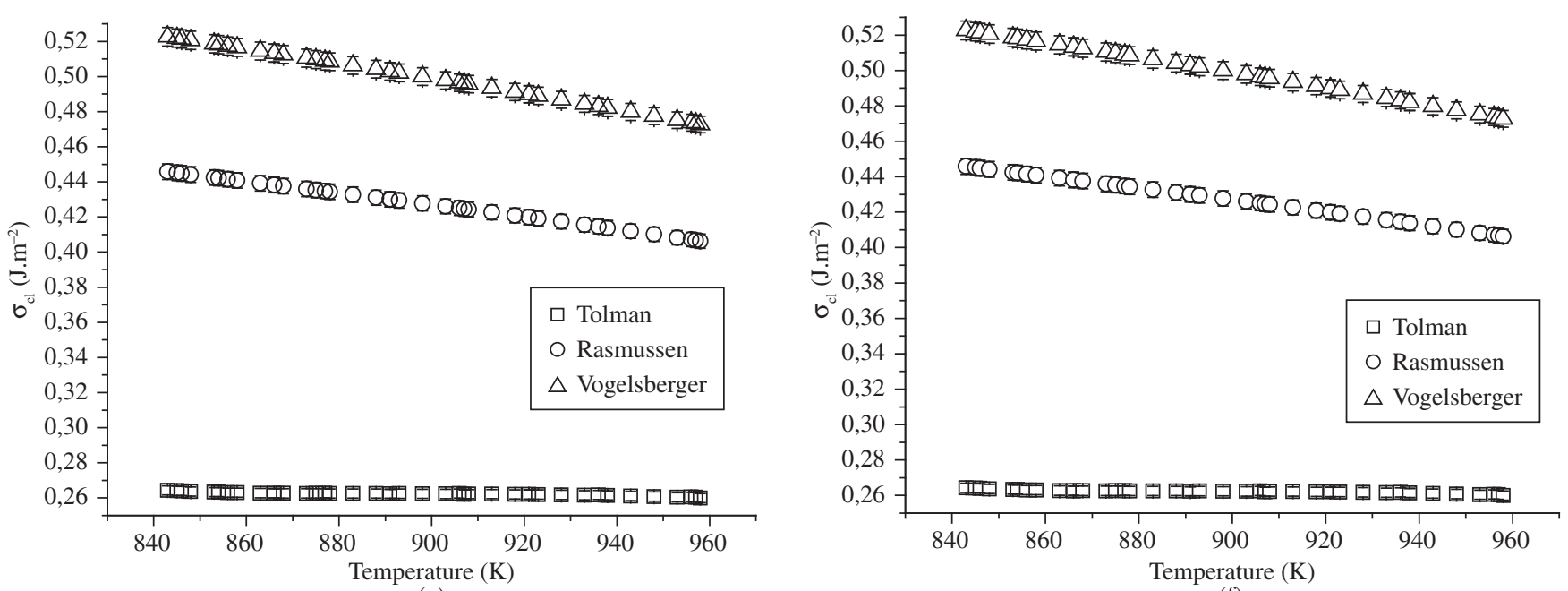

(e)

Figure 3. Crystal-melt surface energy as a function of the temperature calculated for a $\mathrm{N}_{1} \mathrm{C}_{2} \mathrm{~S}_{3}$ glass, using viscosity data and the equations proposed by Tolman $(\square)$, Rasmussen (o) and Vogelsberger $(\triangle)$. The $\delta$ values used were: a) 0 ; b) $2.95 \times 10^{-10} \mathrm{~m}$; c) $4.5 \times 10^{-10} \mathrm{~m}$; d) $7,5 \times 10^{-10} \mathrm{~m}$; e) $8.65 \times 10^{-10} \mathrm{~m}$; f) $10^{-9} \mathrm{~m}$. For $\delta=0$, the same values of $\sigma_{\mathrm{cl}}$ were obtained for all decoupling equations. These values are indicated as ( $\star$ ). 
$\mathrm{d} \sigma_{\mathrm{cl}} / \mathrm{dT}$ as a function of $\lambda$ for $\mathrm{LS}_{2}$ and $\mathrm{N}_{1} \mathrm{C}_{2} \mathrm{~S}_{3}$ glasses, respectively. The dotted lines serve to guide the eyes, while the solid lines indicate the values of Tolman's parameter at the point where $\mathrm{d} \sigma_{\mathrm{cl}} / \mathrm{dT}$ becomes negative

From Figures 4 and 5, one can observe that $\mathrm{d} \sigma_{\mathrm{cl}} / \mathrm{dT}$ decreases gradually, becoming negative as $\delta$ increases. Therefore, physically reasonable values of the Tolman parameter can be chosen in such way that a decrease in surface tension is obtained, as predicted by the $\mathrm{CNT}^{14}$.
Nevertheless, one must analyze the physical meaning of the $\sigma_{\mathrm{cl}}$ values, which can done by calculating the ratio of $\sigma_{\mathrm{cl}} / \sigma_{\mathrm{lv}}\left(\sigma_{\mathrm{lv}}\right.$ is the surface energy in the liquid). In line with Stefan ${ }^{23}, \sigma_{\mathrm{cl}} / \sigma_{\mathrm{lv}} \cong \Delta \mathrm{H}_{\mathrm{cl}} / \Delta \mathrm{H}_{\mathrm{lv}}$ $<<1$; where $\Delta \mathrm{H}_{\mathrm{cl}}$ and $\Delta \mathrm{H}_{\mathrm{lv}}$ are, respectively, the melting enthalpy of the crystalline phase and the enthalpy of evaporation. Considering the $\sigma_{\text {lv }}$ measured by Appen ${ }^{26}$ for a $\mathrm{Li}_{2} \mathrm{O}-\mathrm{SiO}_{2}$ glass composition similar to the one investigated here, we obtained a plot of the $\sigma_{\mathrm{cl}} / \sigma_{\mathrm{lv}}$ ratio as a function of $\delta$, as indicated in Figure 6 .

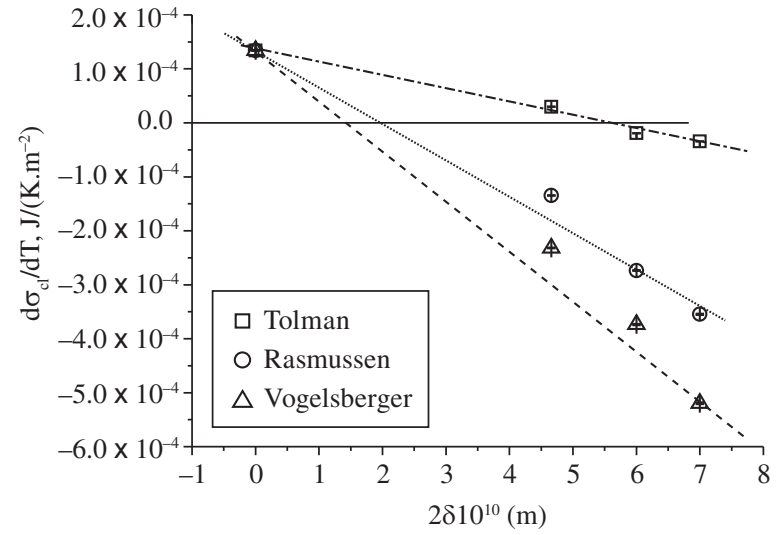

(a)

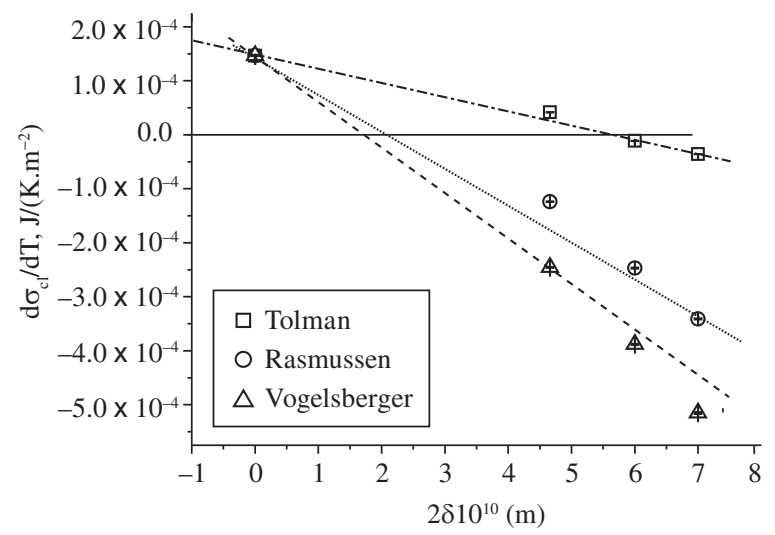

(b)

Figure 4. $\mathrm{d} \sigma_{\mathrm{cl}} / \mathrm{dT}$ as function of $\delta$ for a LS 2 glass using the Tolman, Rasmussen and Vogelsberger expressions, taking into account data of: a) viscosity, and b) induction time.

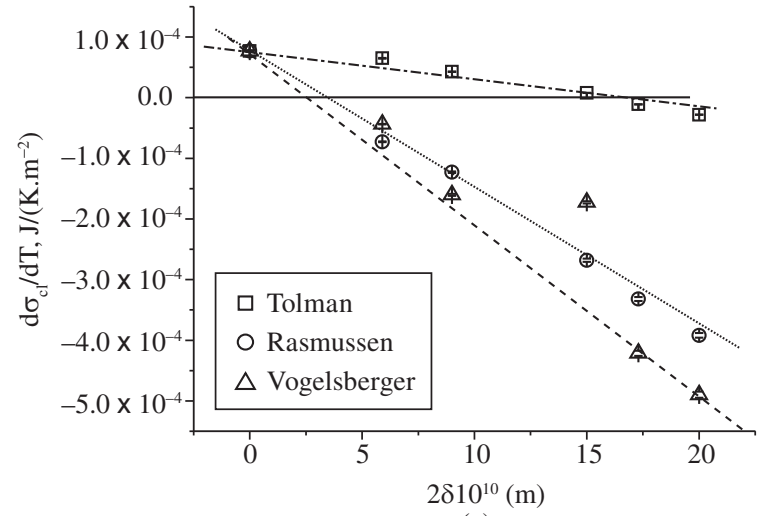

(a)

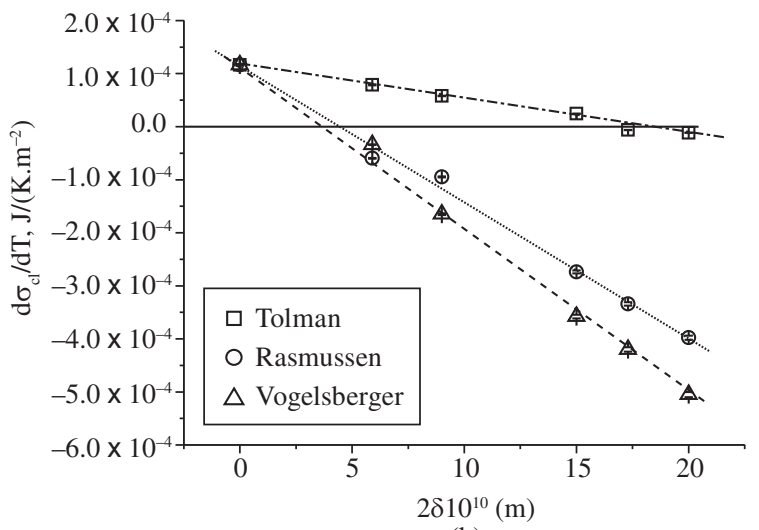

(b)

Figure 5. $\mathrm{d} \sigma_{\mathrm{cl}} / \mathrm{dT}$ as function of $\delta$ for a $\mathrm{N}_{1} \mathrm{C}_{2} \mathrm{~S}_{3}$ glass using the Tolman, Rasmussen and Vogelsberger expressions, taking into account data of: a) viscosity, and b) induction time.

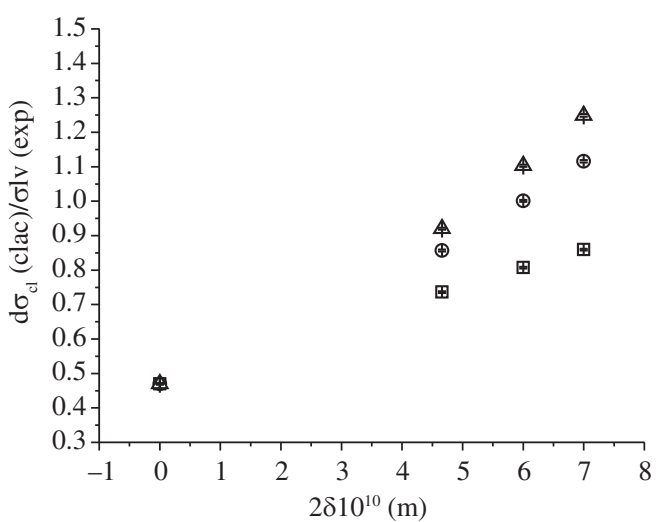

(a)

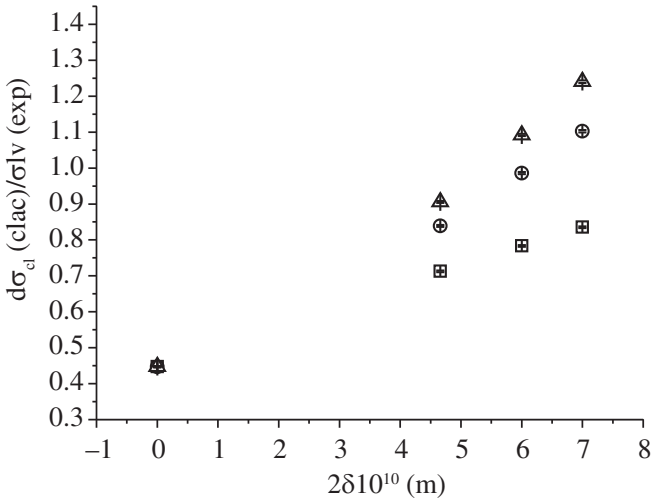

(b)

Figure 6. Comparison of $\sigma_{\mathrm{cl}(\mathrm{calc})} / \sigma_{\mathrm{Iv}(\exp )}$ for the $\mathrm{LS}_{2}$ glass using Tolman, Rasmussen and Vogelsberger expressions. Data of a) viscosity, and b) induction time were employed. 
Taking into account the Rasmussen and Vogelsberger equations, one can observe from Figure 6 that for values of the Tolman's parameter $\left(\delta \geq 6 \times 10^{-10} \mathrm{~m}\right)$ the nuclei/liquid interfacial tension $\left(\sigma_{\mathrm{cl}}\right)$ values are larger than the liquid/vapor ones $\left(\sigma_{\mathrm{lv}}\right)$ i.e. $\sigma_{\mathrm{cl}} / \sigma_{\mathrm{lv}}>>1$. The reason for this result is not clearly understood at this time.

Applying Tolman's equation, one can observe that $\sigma_{\mathrm{cl}} / \sigma_{\mathrm{lv}} \rightarrow 1$. This result also contradicts the one given by Stefan's rule.

\section{Conclusions}

The analysis of the curvature dependence involved the following silicate glasses that exhibit internal nucleation kinetics: $\mathrm{LS}_{2}$ and $\mathrm{N}_{1} \mathrm{C}_{2} \mathrm{~S}_{3}$. In addition to the Tolman equation, the expressions proposed by Rasmussen and Vogelsberger were also applied to decouple the temperature and size parts of surface energy.

The $\sigma_{\mathrm{lc}}(\mathrm{T})$ curves obtained were plotted from experimental data of viscosity, crystal nucleation rate, thermodynamic free energy and induction time. The curves demonstrated that all the decoupling equations used in this work were successfully applied to produce decreasing temperature dependence, in agreement with the results obtained by Fokin \& Zanotto ${ }^{11}$, that applied only the Tolman equation. However, the lowest values of $\sigma_{\mathrm{cl}}(\mathrm{T})$ were obtained with Tolman's expression. This was reinforced by the $\sigma_{\mathrm{cl}} / \sigma_{\mathrm{lv}}$ curves plotted for a $\mathrm{LS}_{2}$ glass.

Despite the lowest values of $\sigma_{\mathrm{lc}}(\mathrm{T})$, a more detailed analysis of the surface energy values was carried out for the $\mathrm{LS}_{2}$ glass using Stefan's rule, which suggests that $\sigma_{\mathrm{cl}} / \sigma_{\mathrm{lv}} \ll 1$. As can be seen in Figure 6, this ratio does not agree with that prediction.

Therefore, it is evident that the assumption of the curvature dependence of surface energy does not suffice, per se, to explain the discrepancy between the experimental and theoretical values of nucleation rates.

\section{Acknowledgements}

The authors gratefully acknowledge the Brazilian research funding agencies CNPQ (\# 620249/2006-4), FINEP, CAPES and FAPEMA for their financial support of this investigation.

\section{References}

1. James PF. Kinetics of crystal nucleation in lithium silicate glasses. Physics and Chemistry of Glasses. 1974; 15(4):95-105.

2. Zanotto ED, James PF. Experimental tests of the classical nucleation theory for glasses. Journal of Non-Crystalline Solids. 1989; 74(2-3):373-394.

3. Rowlands EG, James PF. Analysis of steady-state crystal nucleation rates in glasses: part 2: Further comparison between theory and experiment for lithium disilicate glass. Physics and Chemistry of Glass. 1979; 20(1):1-14.

4. Gonzalez-Oliver CJR. Crystal nucleation and growth in soda-lime-silica glasses [tese]. Sheffield: University of Sheffield; 1979.

5. Fokin VM, Kalinina AM, Filipovich VN. Nucleation in silicate glasses and effect of preliminary heat treatment on it. Journal of Crystal Growth. 1981; 52(part 1):115-121.
6. Cabral Jr AA. Critical cooling rates on silicate glasses that nucleate homogeneously [dissertação]. São Carlos: Federal University of Sao Carlos; 1995.

7. Zanotto ED. Metastable phases in lithium dissilicate glasses. Journal of Non-Crystalline Solids. 1997; 219:42-48.

8. Soares Jr PC, Zanotto ED, Fokin VM, Jain H. TEM and XRD study of early crystallization of lithium disilicate glasses. Journal of Non-Crystalline Solids. 2003; 331(1-3):217-227.

9. Davis MJ, Ihinger PD, Lasaga AC. Influence of water on nucleation kinetics in silicate melt. Journal of Non-Crystalline Solids. 1997; 219:62-69.

10. Fokin VM, Zanotto ED, Schmelzer JWP, Potapov OV. New insights on the thermodynamic barrier for nucleation in glasses: the case of lithium disilicate. Journal of Non-Crystalline Solids. 2005; 351(18):1491-1499.

11. Fokin VM, Zanotto ED. Crystal nucleation in silicate glasses: the temperature and size dependence of crystal/liquid surface energy. Journal of Non-Crystalline Solids. 2000; 265(1-2):105-112.

12. Turnbull D. Formation of crystal nuclei in liquid metals. Journal of Applied Physics. 1950; 21(10):1022-1028.

13. Spaepen F. Homogeneous nucleation and the temperature dependence of the crystal-melt interfacial tension. Solid State Physics. 1994; 47:1-30.

14. Gutzow I, Kashchiev D, Avramov I. Nucleation and crystallization in glass-forming melts: old problems and new questions. Journal of Non-Crystalline Solids. 1985; 73(1-3):477-499.

15. Schmelzer JWP, Gutzow I, Schmelzer Jr. J. Curvature-dependent surface tension and nucleation theory. Journal of Colloid and Interface Science. 1996; 178(2):657-665.

16. Weinberg M, Zanotto ED, Manrich S. Classical nucleation theory with a size dependent interfacial tension: $\mathrm{Li}_{2} \mathrm{O} .2 \mathrm{SiO}_{2}$ crystal nucleation. Physics and Chemistry of Glasses. 1992; 33(3):99-102.

17. Kožlšsek Z. Influence of the curvature dependence of interfacial energy on homogeneous nucleation kinetics. Crystal Research and Technology. 1991; 26(1):3-10.

18. Tolman RC. The effect of droplet size on surface tension. The Journal of Chemical Physics. 1949; 17(3):333-337.

19. Rasmussen DH. Thermodynamics and nucleation phenomena: a set of experimental observations. Journal of Crystal Growth, 1982; 56(1):56-66.

20. Fokin VM. 2006. [Personal Comunication].

21. Schmelzer JWP, Mahnk R. General formulae for the curvature dependence of droplets and bubbles. Journal of the Chemical Society, Faraday Transactions. 1986; 82(1):1413-1420.

22. Fokin VM, Zanotto ED, Yuritsin NS, Schmelzer JWP. Homogeneous crystal nucleation in silicate glasses: a 40 years perspective. Journal of Non-Crystalline Solids. 2006; 352(26-27):2681-2714.

23. Stefan J. Ueber die beziehung zwischen den theorien der capillarität und der verdampfung. Annalen der Physik und Chemie, 1886; 265(12):655.

24. Appen AA, Schischow KA, Kajalowa SS. Die oberflächenspannung von silikatschmelzen. Silikattechnik, 1953; 4(3):104-105. 